DETC2000/MECH-14060

\title{
SOLVING THE KINEMATICS OF PLANAR MECHANISMS BY DIXON DETERMINANT AND A COMPLEX-PLANE FORMULATION
}

\author{
Charles W. Wampler \\ Enterprise Systems Lab \\ General Motors Research and Development \\ Mail Code 480-106-359 \\ 30500 Mound Road \\ Warren, Michigan 48090-9055 \\ Email: charles.w.wampler@gm.com
}

\begin{abstract}
This paper presents a general method for the analysis of any planar mechanism consisting of rigid links connected by revolute and slider joints. The method combines the complex plane formulation of Wampler (1999) with the Dixon determinant procedure of Nielsen and Roth (1999). The result is simple to derive and implement, so in addition to providing numerical solutions, the approach facilitates analytical explorations. The procedure leads to a generalized eigenvalue problem of minimal size. Both input/output problems and the derivation of tracing curve equations are addessed.
\end{abstract}

\section{NOMENCLATURE}

$\ell$ Number of kinematic loops.

$\theta_{j} \quad e^{i \Theta_{j}}$, where $\Theta_{j}$ is an angle, in radians.

$z^{*}$ Complex conjugate of $z$.

\section{INTRODUCTION}

In 1999, two independent solutions were published (Wampler 1999, Nielsen and Roth 1999) for the general input/output problem for planar mechanisms composed of revolute and sliding joints. Wampler also showed how his method is applicable to the analysis of the curve traced by a point fixed to one of the links. These methods are notable for their uniform treatment of all planar mechanisms by algebraic elimination.

Traditionally, elimination-based solutions treat individual mechanism types on a case-by-case basis. Notable ex- amples are the work on various six-bar mechanisms by Freudenstein, et al. (1967), and the solution of the basic seven-link structures (which solve the input/output problem for eight-bar mechanisms) by Innocenti $(1994,1995)$. [It could be argued that the coupler-curve equations obtained by Freudenstein et al. (1967) can also be used to solve the input/output problem for any eight-bar mechanism. See Section 6 below.] A recent contribution (Han, Liao and Liang 2000) solves a particular nine-link structure, which may be viewed as the intersection of a four-bar coupler curve with a Stephenson six-bar curve. Dhingra et al. (1999) give more general procedures for solving planar mechanisms and removing extraneous roots. These principles are illustrated by solving a ten-link input/output problem, equivalent to solving a nine-link structure. The principles are presented as a framework within which one carries out the analysis of any specific mechanism by hand.

An alternative approach is to formulate the problem as a system of polynomial equations and then apply general algorithms for computing a Gröbner basis. Lösch (1995) advocates a two-phase approach: first, a basis is computed over a finite coefficient field, and then, the critical path through algorithm is retraced using multiple-precision floating point arithmetic. This is shown to be effective for treating two nine-link planar structures and one eleven-link planar structure. Dhingra et al. (1998) follow the computation of a Gröbner basis with a solution based on a Sylvestertype determinant. The Gröbner basis methods use symbolic computation extensively, and although in theory the 
algorithms always terminate, in practice the memory space and computational time required can be hard to predict.

In contrast to the Gröbner methods, the elimination algorithms by Wampler and by Nielsen and Roth are specifically formulated for the class of problems at hand. The only symbolic manipulation capability needed is the multiplication of monomials, which amounts to adding exponents, so the methods are easy to code without need of a symbolic computation package. The steps of the elimination are explicitly laid out, so that upper bounds on the space and time required are clear.

As an alternative to elimination, there are many numerical approaches to solving planar mechanisms. These range from using Newton's method to seek a solution near an initial guess, to more sophisticated approaches that find all solutions. Among the latter, polynomial continuation has proven very effective. Examples of its application to planar mechanisms can be found in Wampler (1996) and Waldron and Sreenivasen (1996). Shen, Ting and Yang (2000) show that among the 33 basic planar structures having four or fewer loops, 29 can be reduced to one-dimensional numerical searches. A full listing of the variety of numerical methods is beyond the scope of this paper.

The method described in this paper uses the Dixon determinant, as did Nielsen and Roth, but applies the technique to equations formulated by Wampler in the complex plane. By combining features of both predecessors, we obtain the simplest form yet for the resultant. To establish the proper context of the new method, we briefly outline each of the predecessors. Then we proceed to describe the new method, show its properties, and verify its application to an eight-link mechanism.

\section{FORMULATION OF THE LOOP EQUATIONS}

The starting point for both predecessors (Wampler 1999, Nielsen and Roth 1999) is to reference the rotation angles of all links to a fixed, ground coordinate system. The basic equations are obtained by setting the sum of the vectors around each independent loop of the mechanism equal to zero. Wampler formulates these vectors in the complex plane, so that denoting the angle of link $j$ as $\Theta_{j}$, one has $\ell$ loop equations of the form

$$
c_{k 0}+\sum_{j=1}^{2 \ell} c_{k j} e^{i \Theta_{j}}=0, \quad k=1, \ldots, \ell
$$

Here, $i$ is the imaginary unit $\sqrt{-1}$, the $c_{k j}$ are complex numbers describing the shapes of the links, and link 0 is assumed to be the fixed link. We have assumed that the input an- gle is given and incorporated into the leading constants $c_{k 0}$, leaving only the unknown angles in the summation.

The formulation used by Nielsen and Roth can be obtained by taking the real and imaginary parts of Eqs. (1), thus obtaining two equations for each loop. These are linear in the sine and cosine of the angles and have real coefficients:

$$
\alpha_{k}+\sum_{j=1}^{2 \ell} \beta_{k j} \cos \Theta_{j}+\sum_{j=1}^{2 \ell} \gamma_{k j} \sin \Theta_{j}=0, \quad k=1, \ldots, 2 \ell
$$

Here, $\alpha_{k}, \beta_{k j}$ and $\gamma_{k j}$ are real quantities that depend only on the shape of the links. We call Eqs. (2) a Cartesian formulation of the problem, because the real and imaginary parts correspond to the $X$ and $Y$ Cartesian coordinate directions, respectively. In contrast, Wampler adopts what may be called an isotropic formulation, in which one introduces the variables

$$
\theta_{j}=e^{i \Theta_{j}}, \quad j=1, \ldots, 2 \ell,
$$

so that the loop equations and their complex conjugates may be written as

$$
\begin{aligned}
c_{k 0}+\sum_{j=1}^{2 \ell} c_{k j} \theta_{j} & =0, \\
c_{k 0}^{*}+\sum_{j=1}^{2 \ell} c_{k j}^{*} \theta_{j}^{-1} & =0, \quad k=1, \ldots, \ell .
\end{aligned}
$$

\subsection{Example: Double Butterfly Linkage}

To facilitate direct comparison to previous work, we consider the same example as Nielsen and Roth (1999): a double butterfly linkage. This type of mechanism was studied earlier by Waldron and Sreenivasen (1996), using continuation methods. As shown in Fig. 1, the linkage has three independent loops. We may choose to write each loopclosure equation by leaving ground via link 7 and returning via paths that traverse links 4,5 , and 3 , respectively, to get

$$
\begin{aligned}
& f_{1}: a_{7} \theta_{7}+b_{2} e^{i \gamma_{2}} \theta_{2}-a_{4} \theta_{4}-a_{6} \theta_{6}+a_{0} e^{-i \gamma_{0}}=0 \\
& f_{2}: a_{7} \theta_{7}+a_{2} \theta_{2}+a_{1} \theta_{1}-a_{5} \theta_{5}+b_{0}=0 \\
& f_{3}: a_{7} \theta_{7}+a_{2} \theta_{2}+b_{1} e^{i \gamma_{1}} \theta_{1}-a_{3} \theta_{3}-b_{6} e^{i \gamma_{6}} \theta_{6}+a_{0} e^{-i \gamma_{0}}=0
\end{aligned}
$$

For each of these, there is a corresponding conjugate loop equation as follows.

$$
f_{1}^{*}: a_{7} \theta_{7}^{-1}+b_{2} e^{-i \gamma_{2}} \theta_{2}^{-1}-a_{4} \theta_{4}^{-1}-a_{6} \theta_{6}^{-1}+a_{0} e^{i \gamma_{0}}=0
$$




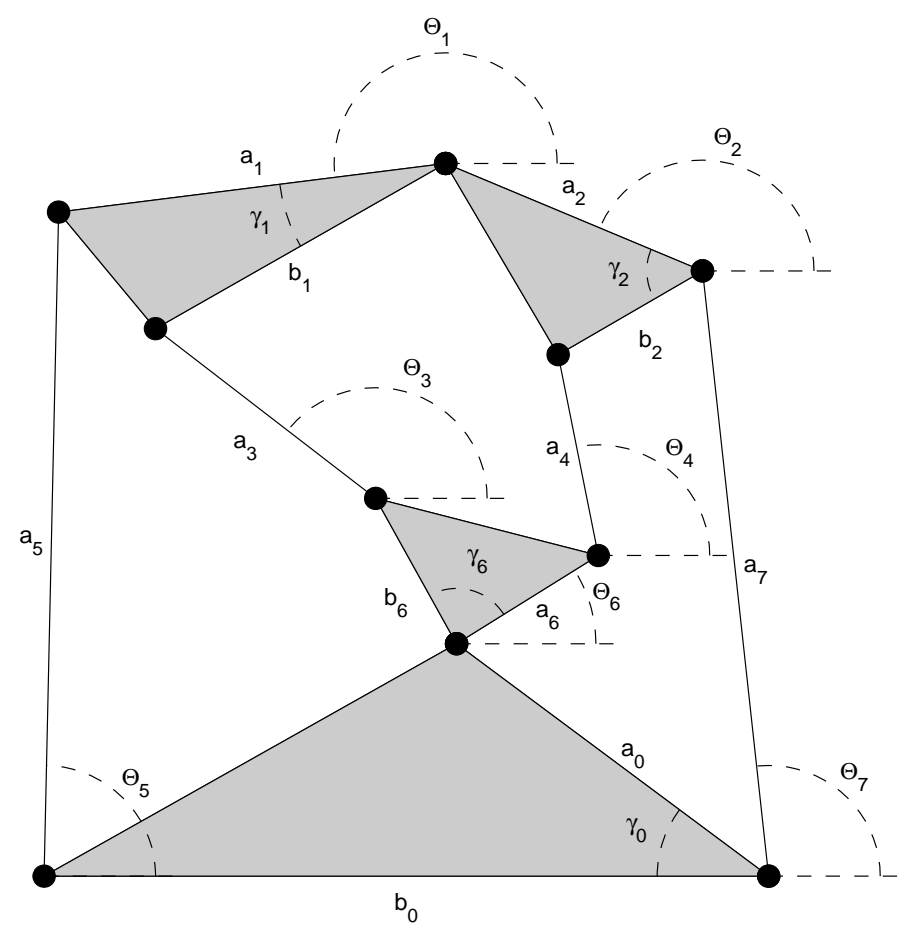

Figure 1. Double-butterfly mechanism

$$
\begin{aligned}
f_{2}^{*}: & a_{7} \theta_{7}^{-1}+a_{2} \theta_{2}^{-1}+a_{1} \theta_{1}^{-1}-a_{5} \theta_{5}^{-1}+b_{0}=0 \\
f_{3}^{*}: a_{7} \theta_{7}^{-1}+a_{2} \theta_{2}^{-1}+b_{1} e^{-i \gamma_{1}} \theta_{1}^{-1}-a_{3} \theta_{3}^{-1} & \\
& -b_{6} e^{-i \gamma_{6}} \theta_{6}^{-1}+a_{0} e^{i \gamma_{0}}=0
\end{aligned}
$$

\section{REVIEW OF RELATED METHODS}

To set the current work in proper context, we briefly review the two previously-published methods that are most closely related.

\subsection{Wampler's Sylvester-type Eliminant}

The crux of a Sylvester-type elimination procedure is to generate auxiliary equations by multiplying the original equations by various monomials. The goal is to make the total number of equations equal to the total number of monomials appearing in the equations. Wampler (1999) provides a simple prescription to meet this criterion. The first step is to use the linear Eqs. (4) and the identities $\theta_{j} \theta_{j}^{-1}=1$, $j=\ell+1, \ldots, 2 \ell$, to obtain $\ell$ equations wherein the $\theta_{j}$ and $\theta_{j}^{-1}, j=1, \ldots, \ell$, appear bilinearly. One variable, say $\theta_{\ell}$, is suppressed (considered to be part of the coefficients of the equations). Then, each equation is multiplied by certain monomials in $\theta_{j}$ to get $(\ell+1)\left(\begin{array}{c}2 \ell-1 \\ \ell\end{array}\right)$ sparse equations in the same number of monomials. This yields a system that can be written in matrix form as

$$
Q \mathbf{m}=\left(Q_{1}+Q_{2} \theta_{\ell}\right) \mathbf{m}=0 .
$$

where $Q_{1}$ and $Q_{2}$ are square with sparse complex entries and $\mathbf{m}$ is a column vector of monomials. The entries in $Q$ are just the coefficients of the bilinear equations redistributed according to the result of multiplication by a monomial. Although the initial size of these matrices is large, at most $\left(\begin{array}{c}2 \ell \\ \ell\end{array}\right)$ columns of $Q_{2}$ are nonzero, so sparse linear factorization techniques can be used to reduce the problem to this smaller size. Then, the solutions for $\theta_{\ell}$ are found using standard routines for the generalized eigenvalue problem, while all other joint values can be found in the corresponding eigenvectors. Since the whole procedure comes down to distributing the coefficients in a certain pattern in a sparse matrix followed by the application of standard linear algebra routines, the method is very easy to program.

The main deficiency of this method is that, for all but the simplest mechanisms, it introduces some extraneous roots. These are easily detected numerically, since the extraneous roots have one or more of the $\theta_{j}$ equal to zero. However, it would be preferable not to include such roots, both to keep the eigenvalue problem smaller and to provide the actual resultant for the problem, which can be useful in further analysis. A more minor criticism of the approach is that it uses complex numbers throughout, which are not supported in some computer languages.

\subsection{Nielsen and Roth's Dixon Determinant Method}

The Dixon determinant is a method of finding the eliminant of a system of polynomial equations. (A description of the general approach appears in the next section.) Nielsen and Roth (1999) adapt this method in an ingenious way to handle their initial equations, which are trigonometric, not polynomial, being linear sine-cosine equations in the angles $\Theta_{1}, \ldots, \Theta_{2 \ell}$. Nonetheless, through a series of trigonometric manipulations, they derive an equation of the form

$$
\left(A \cos \Theta_{2 \ell}+B \sin \Theta_{2 \ell}+C\right) \mathbf{m}=0,
$$

where $A, B, C$ are square constant matrices and $\mathbf{m}$ is column of sines and cosines involving sums and differences of the half-angles of $\Theta_{1}, \ldots, \Theta_{2 \ell-1}$. The next step in the solution method is to use tangent-of-the-half-angle formulas to rewrite Eq. (6) as a polynomial function of $t=\tan \left(\Theta_{2 \ell} / 2\right)$, whereupon the solutions to the problem can be found using generalized eigenvalue routines or by expanding the determinant as a single polynomial. Unfortunately, the tangenthalf-angle substitution doubles the degree of the equations, 
and the solution set includes multiple extraneous factors of $t= \pm i$.

It can be seen that Nielsen and Roth's procedure generates a matrix of size at most $2^{2 \ell-1}$ and hence the eigenvalue problem is size $2^{2 \ell}$. This size is larger than the size of $\left(\begin{array}{c}2 \ell \\ \ell\end{array}\right)$ obtained by Wampler's Sylvester-type eliminant. However, the bound on the size for Nielsen/Roth is loose: the size obtained in actual examples is smaller due to sparsity in the sine-cosine equations.

If matrices $A, B, C$ in Eq.(6) were general, there would be no roots of the special type $t= \pm i$, hence there must be some hidden internal structure to the matrices. A feature of the procedure developed below is that all such structure is clearly revealed, and as a consequence, the size of the eigenvalue problem is kept to a minimum.

\section{THE DIXON DETERMINANT}

The basic technique of both the current paper and the method of Nielsen and Roth is the Dixon determinant (Dixon 1909), which is formulated as follows. Given $N$ polynomials, $f_{1}, \ldots, f_{N}$ in $N-1$ variables $\theta_{1}, \ldots, \theta_{N-1}$, one introduces new variables $\alpha_{1}, \ldots, \alpha_{N-1}$ and forms the determinant

$$
\Delta=\left|\begin{array}{ccc}
f_{1}\left(\theta_{1}, \theta_{2}, \ldots, \theta_{N-1}\right) & \ldots & f_{N}\left(\theta_{1}, \theta_{2}, \ldots, \theta_{N-1}\right) \\
f_{1}\left(\alpha_{1}, \theta_{2}, \ldots, \theta_{N-1}\right) & \ldots & f_{N}\left(\alpha_{1}, \theta_{2}, \ldots, \theta_{N-1}\right) \\
\ldots & & \ldots \\
f_{1}\left(\alpha_{1}, \alpha_{2}, \ldots, \alpha_{N-1}\right) & \ldots & f_{N}\left(\alpha_{1}, \alpha_{2}, \ldots, \alpha_{N-1}\right)
\end{array}\right|
$$

In the $i$ th row of this equation, variables $\theta_{1}, \ldots, \theta_{i-1}$ are replaced by $\alpha_{1}, \ldots, \alpha_{i-1}$. If for any $i$ we let $\theta_{i}=\alpha_{i}$, then row $i$ and row $i+1$ will be identical and so the determinant is zero. Cancelling out such factors, one obtains the Dixon polynomial

$$
\delta\left(\theta_{1}, \ldots, \theta_{N-1}, \alpha_{1}, \ldots, \alpha_{N-1}\right)=\Delta / \prod_{i=1}^{N-1}\left(\theta_{i}-\alpha_{i}\right)
$$

When this determinant is expanded and like terms collected, it can be put into the form $\delta=\mathbf{a} W \mathbf{t}$, where $\mathbf{a}$ is a row vector of monomials in the $\alpha_{i}$ variables, $\mathbf{t}$ is a column vector of monomials in the variables $\theta_{i}$, and $W$ is a function of the coefficients of $f_{1}, \ldots, f_{N}$. It is clear that for a common solution of the original equations, the first row of the determinant is zero, so $\delta$ must also be zero. Moreover, this will be true for arbitrary values of the auxiliary variables $\alpha_{i}$. Consequently, solutions must satisfy the matrix equation

$$
W \mathbf{t}=0 .
$$

It happens that $W$ is square, so a necessary condition that $f_{1}, \ldots, f_{N}$ have a common root is $\operatorname{det}(W)=0$.

Notice that the procedure as just described has one more equation $f_{i}$ than unknown $\theta_{i}$. This does not preclude the possibility that the coefficients of the polynomials $f_{i}$ might depend on an additional variable $\theta_{N}$, which will then appear in the eliminant matrix. This is the method Dixon developed for solving polynomial equations.

\section{THE NEW METHOD}

We proceed by applying Dixon's method to Eqs (4), formulated in the complex plane. This can be done in a straightforward manner, avoiding the complicated trigonometric maneuvers that are at the heart of the Nielsen/Roth method. The result is an eliminant for the problem in simple form.

\section{$5.1 \quad$ Forming the determinant}

Without loss of generality, we may renumber the links so that the output is the final link, $2 \ell$. We suppress the associated rotational variable, which for notational convenience we rename as $x=\theta_{2 \ell}$. Then, following Eq. (7), we form the Dixon determinant $\Delta$, placing the first loop equation and its conjugate in the first two columns, and likewise, forming successive pairs of columns from the remaining loop equations. The determinant $\Delta$ is unchanged if we subtract each row from the one above it, that is, replace each row $i$, for $i=1, \ldots, 2 \ell-1$, with row $i$ minus row $i+1$. After this modification, the $k$ th loop generates a pair of columns in the Dixon determinant as

$$
\left[\begin{array}{cc}
c_{k 1}\left(\theta_{1}-\alpha_{1}\right) & c_{k 1}^{*}\left(\theta_{1}^{-1}-\alpha_{1}^{-1}\right) \\
c_{k 2}\left(\theta_{2}-\alpha_{2}\right) & c_{k 2}^{*}\left(\theta_{2}^{-1}-\alpha_{2}^{-1}\right) \\
\vdots & \vdots \\
c_{k, 2 \ell-1}\left(\theta_{2 \ell-1}-\alpha_{2 \ell-1}\right) & c_{k, 2 \ell-1}^{*}\left(\theta_{2 \ell-1}^{-1}-\theta_{2 \ell-1}^{-1}\right) \\
c_{k 0}+c_{k, 2 \ell} x+\sum_{j=1}^{2 \ell-1} c_{k j} \alpha_{j} & c_{k 0}^{*}+c_{k, 2 \ell}^{*} x^{-1}+\sum_{j=1}^{2 \ell-1} c_{k j}^{*} \alpha_{j}^{-1}
\end{array}\right]
$$

There are $\ell$ such pairs of columns in the $2 \ell \times 2 \ell$ matrix.

This determinant contains roots of the form $\theta_{j}=\alpha_{j}$, which must be removed. This could be done either by factoring out $\left(\theta_{j}-\alpha_{j}\right)$ or $\left(\theta_{j}^{-1}-\alpha_{j}^{-1}\right)$. Both accomplish exactly the same thing, but the latter makes the writing a little simpler, so we choose it. Recall that rescaling a row of a matrix rescales the determinant by the same factor. Noting that $(t-a)=-t a\left(t^{-1}-a^{-1}\right)$, we rescale row $j$ by dividing through by $\left(\theta_{j}^{-1}-\alpha_{j}^{-1}\right)$ to get for loop $k$ the pair of columns 
$\left(\begin{array}{ll}V_{2 k-1} & V_{2 k}\end{array}\right)$ equal to

$\left[\begin{array}{cc}-c_{k 1} \theta_{1} \alpha_{1} & c_{k 1}^{*} \\ -c_{k 2} \theta_{2} \alpha_{2} & c_{k 2}^{*} \\ \vdots & \vdots \\ -c_{k, 2 \ell-1} \theta_{2 \ell-1} \alpha_{2 \ell-1} & c_{k, 2 \ell-1}^{*} \\ c_{k 0}+c_{k, 2 \ell} x+\sum_{j=1}^{2 \ell-1} c_{k j} \alpha_{j} & c_{k 0}^{*}+c_{k, 2 \ell}^{*} x^{-1}+\sum_{j=1}^{2 \ell-1} c_{k j}^{*} \alpha_{j}^{-1}\end{array}\right]$

All of these columns may be gathered into one $2 \ell \times 2 \ell$ matrix $V=\left(\begin{array}{lll}V_{1} & \cdots & V_{2 \ell}\end{array}\right)$, whereupon the Dixon polynomial can be written as

$$
\delta=\operatorname{det}(V)
$$

\subsection{Expanding the determinant}

The next step is to expand the Dixon polynomial and collect terms. The determinant of a $n \times n$ matrix is a sum of terms, each a product of $n$ elements taken one from each row and column, with the appropriate sign attached. From this, it can be seen that the monomials that appear in $\mathbf{t}$ of Eq.(9) have either degree $\ell-1$ or $\ell$, since by Eqs. $(11,12)$ each of the odd columns in $V$ must contribute a factor of $\theta_{i}$ (some $i$ ), unless its bottom element is chosen. But the bottom element can be chosen from at most one column. Moreover, the $\theta_{i}$ 's in such a monomial must all be distinct, since $\theta_{k}$ only appears in row $k$. A similar statement holds for the monomials in the $\alpha_{i}$, which is most easily seen by rewriting $\delta$, starting by subtracting each row from its successor in $\Delta$. This results in columns similar to Eq.(11) with the roles of $\theta_{i}$ and $\alpha_{i}$ interchanged.

It is beneficial to organize the monomials in a certain way. First, find all the combinations of choosing $\ell-1$ distinct variables from the set $\left\{\theta_{1}, \ldots, \theta_{2 \ell-1}\right\}$. The number of such monomials is, by definition, the binomial coefficient $\left(\begin{array}{c}2 \ell-1 \\ \ell-1\end{array}\right)$. Order these in any arbitrary way in a column vector $\mathbf{t}_{1}$. For each monomial in $\mathbf{t}_{1}$, we may say that its complement is a monomial of degree $\ell$, given by choosing the complementary set of variables. For example consider the case of a 3-loop mechanism, so that, disregarding the suppressed variable $x=\theta_{6}$, the variables in question are $\theta_{1}, \ldots, \theta_{5}$. Then, for one of the monomials in $\mathbf{t}_{1}$, say $\theta_{2} \theta_{5}$, the complementary monomial in $\mathbf{t}_{2}$ is $\theta_{1} \theta_{3} \theta_{4}$. We may define row vectors $\mathbf{a}_{1}$ and $\mathbf{a}_{2}$ in a similar fashion, such that the monomial obtained by replacing each $\theta_{i}$ with $\alpha_{i}$ is in the same location. The abbreviated notations $\mathbf{t}=\left(\begin{array}{ll}\mathbf{t}_{1}^{T} & \mathbf{t}_{2}^{T}\end{array}\right)^{T}$ and $\mathbf{a}=\left(\begin{array}{ll}\mathbf{a}_{1} & \mathbf{a}_{2}\end{array}\right)$ are useful. With these definitions, we arrive at the main result, as follows.
Main Result. The Dixon polynomial for Eqs.(4) may be written as

$$
\begin{aligned}
\delta & =\mathbf{a} W \mathbf{t} \\
& =\left(\begin{array}{ll}
\mathbf{a}_{1} & \mathbf{a}_{2}
\end{array}\right)\left(\begin{array}{cc}
D_{1} x+D_{2} & A^{T} \\
A & s\left(D_{1}^{*} x^{-1}+D_{2}^{*}\right)
\end{array}\right)\left(\begin{array}{l}
\mathbf{t}_{1} \\
\mathbf{t}_{2}
\end{array}\right)=0,
\end{aligned}
$$

where $s=(-1)^{\ell-1}, D_{1}$ and $D_{2}$ are diagonal and the elements of $A$ obey the relation $a_{i j}=s a_{j i}^{*}$. Moreover, each column of $A$ contains at most $\ell$ nonzero elements.

The sparsity of $W$ can be seen from the row-expansion algorithm for computing a determinant. Recall that the determinant of an $n \times n$ matrix $V$ can be written as

$$
\operatorname{det}(V)=\sum_{i=1}^{n}(-1)^{r+i} v_{r i} \operatorname{det}\left(V_{r i}\right)
$$

where $v_{r i}$ is the element in the $r$ th row and $i$ th column of $V$, and $V_{r i}$ is $V$ with the $r$ th row and $i$ th column struck out. In our particular case, by selecting $r=n=2 \ell$ as the bottom row, $\operatorname{det}\left(V_{n i}\right)$ is a sum of monomials in which only factors of the form $\alpha_{j} \theta_{j}$ appear, containing $\ell-1$ such factors when $i$ is odd and $\ell$ such factors when $i$ is even. Since $v_{n i}$ is a sum of terms, we must consider what happens when $\operatorname{det}\left(V_{n i}\right)$ is multiplied by each of these. For $i$ odd, each term in $v_{n i}$ is a coefficient times one of the following: $\left\{1, x, \alpha_{1}, \ldots, \alpha_{2 \ell-1}\right\}$. The factors $x$ and 1 place a contribution on the diagonal in $D_{1}$ and $D_{2}$, respectively. The factors in $\alpha_{k}$ push the contribution down the same column into the lower-left sub-matrix $A$. But only the $\alpha_{k}$ that do not appear in $\operatorname{det}\left(V_{n i}\right)$ can contribute, as argued in the first paragraph of this section, so only $\ell$ elements in the column of $A$ can be nonzero. Similarly, even values of $i$ give entries in the right-hand blocks of $W$. Since the coefficients in the even columns of $V$ are the conjugates of those in the odd columns, entries in the righthand blocks of $W$ are conjugates of those on the left, with appropriate adjustment of the sign. The symmetry within $A$ is due to the fact that each coefficient $c_{k i}$ appears twice in column $i$ : once in row $i$ and once in the bottom row. A rigorous analysis is tedious, so we opt instead to illustrate the phenomenon by an example in Appendix A.

The row-expansion algorithm just discussed can be implemented directly as a computational tool. It is sufficient to expand only the odd elements along the bottom row to compute the left-hand side of $W$. The right-hand side is then easily derived by symmetry, thus saving half the work. The work might be reduced even further if the symmetry within $A$ were used to advantage, although an easy method to do so is not immediately apparent. 
If any column in $\delta$ is zero, we may of course omit it. Because of the symmetry relations, if the column is in the left-hand side of $W$, then there is a corresponding zero column in the right-hand side. Moreover, there must also be corresponding rows that are zero. When these are all omitted, the matrix is still square with its symmetries intact. Consequently, although the size of the matrix is at most $\left(\begin{array}{c}2 \ell \\ \ell\end{array}\right)$, it is often some multiple of two less than this number.

A computational alternative to row expansion of the determinant is to evaluate the elements of $W$ by fitting. That is, for sample numerical values of $\theta_{1}, \ldots, \theta_{2 \ell}$ and $\alpha_{1}, \ldots, \alpha_{2 \ell-1}$, the value of $\delta$ can be computed from Eq.(12). On the other hand, Eq.(13) shows that $\delta$ is a linear function of the elements in $D_{1}, D_{2}$, and $A$. So after collecting enough sample values of $\delta$, these elements can be found by solution of a set of linear equations. At each sample, $V$ is just a matrix of numerical values, so its determinant is efficiently evaluated by a triangular decomposition of $V$, rather than by row expansion. The minimal number of samples needed is the number of distinct non-zero elements $D_{1}, D_{2}$, and $A$, so the sparsity and symmetries of these matrices greatly reduce the amount of computation needed.

\subsection{Solving for the output variable}

Since Eq.(13) must hold for arbitrary values of $\alpha_{1}, \ldots, \alpha_{2 \ell-1}$, we have the matrix equation

$$
W \mathbf{t}=\left(\begin{array}{cc}
D_{1} x+D_{2} & A^{T} \\
A & s\left(D_{1}^{*} x^{-1}+D_{2}^{*}\right)
\end{array}\right)\left(\begin{array}{l}
\mathbf{t}_{1} \\
\mathbf{t}_{2}
\end{array}\right)=0 .
$$

This can be converted to a standard generalized eigenvalue problem by multiplying the lower block of equations by $x$ to get

$$
\left[\left(\begin{array}{cc}
D_{1} & 0 \\
A & s D_{2}^{*}
\end{array}\right) x+\left(\begin{array}{cc}
D_{2} & A^{T} \\
0 & s D_{1}^{*}
\end{array}\right)\right]\left(\begin{array}{l}
\mathbf{t}_{1} \\
\mathbf{t}_{2}
\end{array}\right)=0
$$

Note that the two matrices in the eigenvalue problem are triangular, so that the rank is the number of non-zero elements on the diagonal. Considering the matrix which multiplies $x$, one sees that for each zero element on the diagonal of $D_{1}$ or $D_{2}^{*}$, there is an eigenvalue of $x$ at infinity, which is of no interest. The same zeros will show up in the second matrix and lead to trivial eigenvalues of $x=0$, also not of interest. Accordingly, the number of finite, nonzero eigenvalues decreases by two for each such zero element on the diagonal. In such cases, the size of the eigenvalue problem can be reduced by linear methods.

A consequence of the form of the equations is that if $x$ is a root, then $y=1 / x^{*}$ is also a root. This is easily seen by writing the conjugate of each equation in Eq.(15). For roots of unit magnitude, $x x^{*}=1$, this gives $y=x$. These are the "real" roots of the equation, since we are looking for unit-magnitude rotations. All other roots appear in pairs. These pairs are the equivalent of complex-conjugate root pairs that appear in equations with real coefficients.

Instead of solving the generalized eigenvalue problem, one may set the determinant of $W$ to zero. Although this is not a wise choice numerically, it does give the resultant of the problem in the form

$$
\begin{aligned}
k_{d} x^{d}+ & k_{d-1} x^{d-1}+\cdots+k_{1} x+k_{0}+ \\
& k_{1}^{*} x^{-1}+\cdots+k_{d-1}^{*} x^{-d+1}+k_{d}^{*} x^{-d}=0,
\end{aligned}
$$

where $2 d$ is the number of finite, non-zero roots and $k_{0}$ is real.

\subsection{Backsolving}

If the output variable is obtained using the generalized eigenvalue approach, the remaining rotations are found easily from the eigenvectors. The procedure is most simply described with an example. Suppose the mechanism in question has three loops. Then, one of the monomials in $\mathbf{t}_{1}$ is $\theta_{1} \theta_{2}$ and one of the monomials in $\mathbf{t}_{2}$ is $\theta_{1} \theta_{2} \theta_{3}$. Since eigenvectors are only defined up to scale, the numerical routine returns elements $\mu \theta_{1} \theta_{2}$ and $\mu \theta_{1} \theta_{2} \theta_{3}$, for some unknown scaling factor $\mu$. So, one finds the value of $\theta_{3}$ by division: $\theta_{3}=\left(\mu \theta_{1} \theta_{2} \theta_{3}\right) /\left(\mu \theta_{1} \theta_{2}\right)$. Solutions for all of the variables can be found in the same manner.

\subsection{Sliding Joints}

In the formulation of Wampler (1999), if a loop of a mechanism includes a sliding joint, the corresponding loop equation includes a term of the form $s_{i} \theta_{j}$, where $s_{i}$ is the sliding distance (a real number) of link $i$ and $\theta_{j}$ is the rotation of the prior link $j$. The conjugate loop equation contains a corresponding term $s_{i} \theta_{j}^{-1}$, because conjugation of a real number does not alter it. Because both $s_{i}$ and $\theta_{j}$ still appear linearly, the factors $\left(\theta_{j}-\alpha_{j}\right)$ and $\left(s_{i}-\alpha_{i}\right)$ are readily removed from the Dixon determinant. Accordingly, the methodology proceeds in much the same way as for the case of all rotational joints, although the exact form of $\delta$ will vary from that given in Eq.(13).

\subsection{Relative Angles}

We have used a formulation in terms of absolute angles. One could, however, develop a similar procedure using relative angles. For example, suppose that in Fig.1, the rotation of link 1 is measured relative to link 2 , so that the absolute 
rotation of the link is $\Theta_{1}+\Theta_{2}$. Then, a monomial of the form $\theta_{1} \theta_{2}$ would appear in the loop equations instead of $\theta_{1}$. Similar to the case of sliding joints, the individual variables, $\theta_{1}$ and $\theta_{2}$, still appear linearly, so the application of Dixon's method is again straightforward.

\subsection{Example Solution}

The double-butterfly mechanism of Fig. 1 will be used as an example of application of the foregoing method. In particular, assume that $\theta_{7}$ is the input and $x=\theta_{6}$ is the output. Since the mechanism has three loops, at most $\left(\begin{array}{l}5 \\ 2\end{array}\right)=10$ monomials appear in $\mathbf{t}_{1}$, being all the monomials formed by choosing two distinct variables from $\left\{\theta_{1}, \ldots, \theta_{5}\right\}$. However, since not every variable appears in every loop equation, one of these monomials, namely $\theta_{2} \theta_{4}$, does not appear in $\delta$. By the same token, its complement $\theta_{1} \theta_{3} \theta_{5}$ does not appear in $\mathbf{t}_{2}$. Thus, the size of matrix $W$ in Eq.(13) is $18 \times 18$. Moreover, the element of $D_{1}$ which multiplies $\theta_{3} \theta_{4}$ is zero. This same zero appears again on the diagonal in $D_{1}^{*}$. From these observations, it is clear that the input/output problem with $\theta_{6}$ as output has 16 nonzero, finite solutions. The combinatorics involved are simple enough to carry out by hand.

When the method is carried out on a numerical example, the existence of zero elements on the diagonal can be used to reduce the eigenvalue problem to size $16 \times 16$. But the efficiency to be gained by this is minor, so we proceed to solve the problem as a generalized eigenvalue problem of size $18 \times 18$. We have tested the method on the same example used by Nielsen and Roth (1999), namely

$$
\begin{array}{lll}
a_{0}=7.0 & b_{0}=13.0 & \gamma_{0}=36.87^{\circ} \\
a_{1}=7.0 & b_{1}=6.0 & \gamma_{1}=22.62^{\circ} \\
a_{2}=5.0 & b_{2}=3.0 & \gamma_{2}=53.13^{\circ} \\
a_{3}=7.0 & a_{4}=9.0 & a_{5}=12.0 \\
a_{6}=3.0 & b_{6}=2.0 & \gamma_{g}=36.87^{\circ} \\
a_{7}=11.0 & & \Theta_{7}=116.2^{\circ}
\end{array}
$$

The general method has been implemented in Matlab, which uses double-precision arithmetic. The generalized eigenvalue problem is solved using the eig command. When applied to this example, the two expected degenerate roots are reported with magnitudes $1.017 \mathrm{e}+32$ and $1.002 \mathrm{e}-16$, which are clearly distinguishable from the nondegenerate roots. The 16 nondegenerate roots are reported in Table 1 , where the output angle $\Theta_{6}$ is computed, in radians, from the rotation variable $\theta_{6}$ as $\Theta_{6}=-i \log \theta_{6}$. Four of the roots are real. All these numerical results are in agreement with Nielsen and Roth.

A direct comparison shows that for this problem Nielsen and Roth's method leads to an eigenvalue problem of size

\begin{tabular}{crr|rrc}
$\theta_{6}$ & $\left|\theta_{6}\right|$ & \multicolumn{1}{c}{$\Theta_{6}$} & $\theta_{6}$ & $\left|\theta_{6}\right|$ & $\Theta_{6}$ \\
\hline$-6.657,12.555 i$ & 14.211 & $2.058,-2.654 i$ & $-0.995,-0.100 i$ & 1.000 & $-3.041,0.000 i$ \\
$-8.083,-7.094 i$ & 10.755 & $-2.421,-2.375 i$ & $-0.773,-0.634 i$ & 1.000 & $-2.455,0.000 i$ \\
$2.100,-1.599 i$ & 2.640 & $-0.651,-0.971 i$ & $-0.646,0.192 i$ & 0.674 & $2.852,0.394 i$ \\
$0.977,1.497 i$ & 1.788 & $0.992,-0.581 i$ & $0.306,0.468 i$ & 0.559 & $0.992,0.581 i$ \\
$-0.583,-1.493 i$ & 1.603 & $-1.943,-0.472 i$ & $-0.227,-0.581 i$ & 0.624 & $-1.943,0.472 i$ \\
$-1.422,0.423 i$ & 1.484 & $2.852,-0.394 i$ & $0.301,-0.229 i$ & 0.379 & $-0.651,0.971 i$ \\
$0.998,-0.067 i$ & 1.000 & $-0.067,0.000 i$ & $-0.070,-0.061 i$ & 0.093 & $-2.421,2.375 i$ \\
$0.553,-0.833 i$ & 1.000 & $-0.985,0.000 i$ & $-0.033,0.062 i$ & 0.070 & $2.058,2.654 i$
\end{tabular}

Table 1 . Solutions to the example problem

36, Wampler's Sylvester-type eliminant leads to size 20 (reducible to 18 by linear factorization methods), and the current method leads to size 18 (reducible to 16 by linear factorization). All give good numerical results on this problem.

\section{TRACING CURVES}

As noted in previous papers (Wampler 1996,1999), there is a close relationship between input/output problems and the curves traced out by points affixed to a mechanism. This relationship is even more strikingly revealed when the method of this paper is applied. Let $p$ be the position of the tracing point. Then, in addition to the loop equations for the mechanism, we may write one more equation expressing $p$ in terms of a chain of links from ground up to the tracing point:

$$
b_{0}+\sum_{i=1}^{2 \ell+1} b_{i} \theta_{i}+p=0
$$

where as in the case of loop equations, the coefficients $b_{0}, \ldots, b_{2 \ell+1}$ are known constants describing the shapes of the links. There is a corresponding conjugate equation

$$
b_{0}^{*}+\sum_{i=1}^{2 \ell+1} b_{i}^{*} \theta_{i}^{-1}+p^{*}=0 .
$$

Now, the method proceeds exactly as before with both $p$ and $p^{*}$ suppressed. The size of the problem is the same as for an input/output mechanism having $\ell+1$ loops, and the only change is that $p$ and $p^{*}$ appear in place of $x$ and $x^{-1}$, respectively. The Dixon polynomial thus becomes

$$
\delta=\left(\begin{array}{ll}
\mathbf{a}_{1} & \mathbf{a}_{2}
\end{array}\right)\left(\begin{array}{cc}
D_{1} p+D_{2} & A^{T} \\
A & s\left(D_{1}^{*} p^{*}+D_{2}^{*}\right)
\end{array}\right)\left(\begin{array}{l}
\mathbf{t}_{1} \\
\mathbf{t}_{2}
\end{array}\right)=0
$$




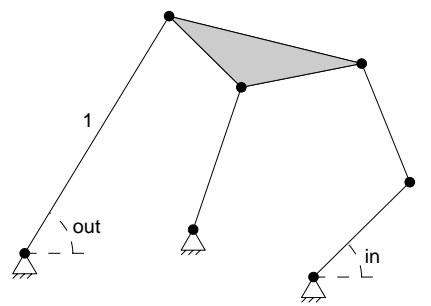

(a)

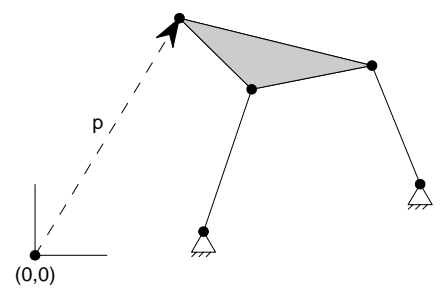

(b)
Figure 2. Input/output and tracing curve equivalence

where the structure of the sub-matrices is exactly as given in Eq.(13). The degree and circularity of the tracing curve are readily gleaned from the number of nonzero elements on the diagonal of $D_{1}$.

The equivalence is illustrated most clearly by considering a particular example. Figure 2a shows an input/output problem for a Stephenson six-bar mechanism, scaled so that the output link is unit length and translated to place the output joint at the origin. Figure $2 \mathrm{~b}$ shows a related fourbar linkage whose tracing point is on the coupler link. We see that the input/output problem is exactly the same as determining points where the coupler curve intersects the unit circle centered at the origin, that is, points where $p p^{*}=1$. This is equivalent to $p^{*}=p^{-1}$, which when substituted into Eq.(21) gives exactly Eq.(13).

\section{CONCLUSIONS}

In this paper, a solution procedure is given for solving input/output problems for planar linkages having revolute or sliding joints. Although inspired by the method of Nielsen and Roth, also based on the Dixon determinant, the method presented in this paper is a significant improvement. The difference in the methods is the use here of loop equations formulated in the complex-plane, which are polynomial, instead of equations in Cartesian coordinates, which are trigonometric. This complex-plane approach gives a resultant matrix that is both sparse and highly structured. The derivation of the matrix is simple and avoids any of the extensive trigonometric manipulations required in a Cartesian approach. When numerical solutions are sought, the complex-plane formulation leads to an eigenvalue problem that is half of the size as that obtained in Cartesian coordinates.
The method also applies to the derivation of the equation for the curve traced out by a point affixed to a planar linkage. The connection between input/output problems and tracing curves is simple and fundamental. In spite of this, outside of Wampler (1996), this relation has seemingly gone unnoticed.

\section{REFERENCES}

Dhingra, A.K., Almadi, A.N., and Kohli, D., 1998, "A Gröbner-Sylvester hybrid method for closed-form displacement analysis of mechanisms," Proc. ASME Des. Eng. Tech. Conf., Sept. 13-16, Atlanta, GA, Paper DETC98/MECH-5969.

Dhingra, A.K., Almadi, A.N., and Kohli, D., 1999, "A framework for closed-form displacement analysis of planar mechanisms," ASME J. Mech. Design, Vol. 121, No. 3, pp. 392-401.

Dixon, A.L., 1909, "The eliminant of three quantics in two independent variables," Proc. London Math. Soc., Ser. 2, Vol. 7, pp. 49-69.

Han, L., Liao, Q., and Liang, C., 2000, "Closed-form displacement analysis for a nine-link Barranov truss or a eight-link Assur group," Mech. Mach. Theory, Vol. 35, No. 3, pp. 379-390.

Innocenti, C., 1994, "Analytical-Form Position Analysis of the 7-Link Assur Kinematic Chain with Four SeriallyConnected Ternary Links", Vol. 116, No. 2, pp. 622-628.

Innocenti, C., 1995a, "Polynomial solution to the position analysis of the 7-link Assur kinematic chain with one quaternary link," Mech. Mach. Theory, Vol. 30, No. 8, pp. 1295-1303.

Lösch, S., 1995, "Parallel redundant manipulators based on open and closed normal Assur Chains," Computational Kinematics, J.-P. Merlet and B. Ravani, eds., Kluwer Academic Publ., Dordrecht, The Netherlands, pp. 251-260.

Nielsen, J., and Roth, B., 1999, "Solving the input/output problem for planar mechanisms," ASME J. Mech. Design, Vol. 121, No. 2, pp. 206-211.

Primrose, E.J.F., Freudenstein, F., and Roth, B., 1967, "Six-bar motion (Parts I-III)," Archive for Rational Mechanics and Analysis, Vol. 24, pp. 22-77.

Shen, H., Ting, K.-L., and Yang, T., 2000, "Configuration analysis of complex multiloop linkages and manipulators," Mech. Mach. Theory, Vol. 35, No. 3, pp. 353-362.

Waldron, K.J. and Sreenivasen, S.V., 1996, "A study of the solvability of the position problem for multi-circuit mechanisms by way of example of the double butterfly linkage," ASME J. Mech. Design, Vol. 118, No. 3, pp. 390-395.

Wampler, C., 1996, "Isotropic coordinates, circularity, and Bezout numbers: planar kinematics from a new per- 
spective," Proc. ASME Des. Eng. Tech. Conf., Aug. 18-22, Irvine, CA, Paper 96-DETC/Mech-1210.

Wampler, C., 1999, "Solving the kinematics of planar mechanisms," ASME J. Mech. Design, Vol. 121, No. 3, pp. 387-391.

\section{A SYMMETRY EXAMPLE}

The symmetries spelled out in Eq.(13) are most easily seen by an example. Consider a general 2-loop mechanism having loop equations

$$
c_{i 0}+c_{i 1} \theta_{1}+c_{i 2} \theta_{2}+c_{i 3} \theta_{3}+c_{i 4} x=0, \quad i=1,2,
$$

where $x=\theta_{4}$ is the variable which we will suppress. Then the Dixon polynomial $\delta$ is expressed in terms of the monomials $\mathbf{t}_{1}=\left\{\theta_{1}, \theta_{2}, \theta_{3}\right\}$ and their complements $\mathbf{t}_{2}=\left\{\theta_{2} \theta_{3}, \theta_{1} \theta_{3}, \theta_{1} \theta_{2}\right\}$, and the corresponding monomials $\mathbf{a}_{1}=\left\{\alpha_{1}, \alpha_{2}, \alpha_{3}\right\}$ and $\mathbf{a}_{2}=\left\{\alpha_{2} \alpha_{3}, \alpha_{1} \alpha_{3}, \alpha_{1} \alpha_{2}\right\}$. For purposes of illustration, it will be sufficient to show how specific terms within this 2-loop example display the claimed symmetries. Note that for $\ell=2$, the sign factor in Eq.(13) is $s=(-1)^{\ell-1}=-1$.

There are two types of symmetry: terms that appear in the diagonal matrices $D_{1}$ or $D_{2}$ and terms that appear in the off-diagonal matrix $A$. Let us first consider terms of the diagonal type. Two such terms are

$$
\left|\begin{array}{cccc}
0 & c_{11}^{*} & 0 & 0 \\
0 & 0 & -c_{22} \alpha_{2} \theta_{2} & 0 \\
0 & 0 & 0 & c_{23}^{*} \\
c_{10} & 0 & 0 & 0
\end{array}\right|,\left|\begin{array}{cccc}
-c_{11} \alpha_{1} \theta_{1} & 0 & 0 & 0 \\
0 & 0 & 0 & c_{22}^{*} \\
0 & 0 & -c_{23} \alpha_{3} \theta_{3} & 0 \\
0 & c_{10}^{*} & 0 & 0
\end{array}\right| .
$$

With the definition $d=c_{10} c_{11}^{*} c_{22} c_{23}^{*}$, these two terms evaluate to $d \alpha_{2} \theta_{2}$ and $-d^{*}\left(\alpha_{1} \alpha_{3}\right)\left(\theta_{1} \theta_{3}\right)$, respectively. These appear in the 2-2 and 5-5 positions in the resultant matrix $W$, and the latter is the negative conjugate of the former, as expected. All other terms on the diagonal follow a similar pattern.

Terms entering the off-diagonal matrix $A$ appear in sets of four, illustrated by the following:

$$
\begin{aligned}
& \left|\begin{array}{cccc}
0 & c_{11}^{*} & 0 & 0 \\
0 & 0 & -c_{22} \alpha_{2} \theta_{2} & 0 \\
0 & 0 & 0 & c_{23}^{*} \\
c_{13} \alpha_{3} & 0 & 0 & 0
\end{array}\right|,\left|\begin{array}{cccc}
0 & c_{11}^{*} & 0 & 0 \\
0 & 0 & -c_{22} \alpha_{2} \theta_{2} & 0 \\
-c_{13} \alpha_{3} \theta_{3} & 0 & 0 & 0 \\
0 & 0 & 0 & c_{23}^{*} \alpha_{3}^{-1}
\end{array}\right|, \\
& \left|\begin{array}{cccc}
-c_{11} \alpha_{1} \theta_{1} & 0 & 0 & 0 \\
0 & 0 & 0 & c_{22}^{*} \\
0 & c_{13}^{*} & 0 & 0 \\
0 & 0 & c_{23} \alpha_{3} & 0
\end{array}\right|,\left|\begin{array}{cccc}
-c_{11} \alpha_{1} \theta_{1} & 0 & 0 & 0 \\
0 & 0 & 0 & c_{22}^{*} \\
0 & 0 & -c_{23} \alpha_{3} \theta_{3} & 0 \\
0 & c_{13}^{*} \alpha_{3}^{-1} & 0 & 0
\end{array}\right| .
\end{aligned}
$$

With $a=c_{13} c_{11}^{*} c_{22} c_{23}^{*}$, these evaluate to $a\left(\alpha_{2} \alpha_{3}\right) \theta_{2}$, $a \alpha_{2}\left(\theta_{2} \theta_{3}\right),-a^{*}\left(\alpha_{1} \alpha_{3}\right) \theta_{1}$, and $-a^{*} \alpha_{1}\left(\theta_{1} \theta_{3}\right)$, respectively. The contributions of these four terms and the two terms described in the previous paragraph appear in the following positions within $W$

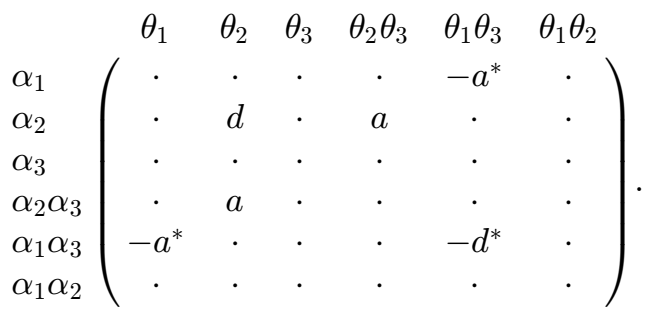

These are seen to obey the expected symmetry relations. 